\title{
Statistical Shape and Appearance Models: Development Towards Improved Osteoporosis Care
}

\author{
Lorenzo Grassi $^{1}$ (D) Sami P. Väänänen ${ }^{2,3} \cdot$ Hanna Isaksson ${ }^{1}$ \\ Accepted: 27 September 2021 / Published online: 13 November 2021 \\ (C) The Author(s) 2021
}

\begin{abstract}
Purpose of Review Statistical models of shape and appearance have increased their popularity since the 1990s and are today highly prevalent in the field of medical image analysis. In this article, we review the recent literature about how statistical models have been applied in the context of osteoporosis and fracture risk estimation.

Recent Findings Recent developments have increased their ability to accurately segment bones, as well as to perform 3D reconstruction and classify bone anatomies, all features of high interest in the field of osteoporosis and fragility fractures diagnosis, prevention, and treatment. An increasing number of studies used statistical models to estimate fracture risk in retrospective case-control cohorts, which is a promising step towards future clinical application.

Summary All the reviewed application areas made considerable steps forward in the past 5-6 years. Heterogeneities in validation hinder a thorough comparison between the different methods and represent one of the future challenges to be addressed to reach clinical implementation.
\end{abstract}

Keywords Osteoporosis $\cdot$ Statistical shape and appearance models $\cdot$ Fracture risk $\cdot$ Femur $\cdot$ Vertebrae $\cdot$ Hip

\section{Introduction}

Osteoporosis is a disease that significantly affects the elderly population. It is characterized by reduced bone mass and altered bone quality, thus resulting in increased bone fragility and fracture risk. About 1 in 3 women and 1 in 5 men above the age of 50 years will suffer from an osteoporotic fracture during their lifetime [1]. The high socioeconomic burden of osteoporotic fractures and the rising incidence [2] call for an improvement of the current standard of care of osteoporosis, spanning from estimation of risk for future fractures (36-72\%

This article is part of the Topical Collection on Imaging

Lorenzo Grassi

lorenzo.grassi@bme.lth.se

1 Department of Biomedical Engineering, Lund University, Box 118, 22100 Lund, Sweden

2 Department of Applied Physics, University of Eastern Finland, Kuopio, Finland

3 Diagnostic Imaging Center, Kuopio University Hospital, Kuopio, Finland of fractures in women with an areal bone mineral density (aBMD) above the threshold for osteoporosis diagnosis [3, 4]) to fracture treatment (40-60\% of subjects with hip fracture do not recover their pre-fracture mobility [5]).

Advances in functional medical imaging hold great promise for improving many areas of osteoporosis standard-of-care by providing more accurate and quantitative methods. In particular, statistical models of shape and appearance [6-8] have assumed an increasingly important role during the past 10 years. This is due to the fact that such models are capable of providing accurate and automatic segmentation of medical images [9] as well as to generate numerical models to quantitatively assess the influence of several parameters on fracture risk and assist in preoperative planning [10].

This review aims to present the most recent applications of statistical models to the field of osteoporosis. A literature search was performed for the most relevant applications of statistical shape and appearance models in the field of osteoporosis during the past 5 years. First, the technical aspects of the methods are summarized, followed by the most recent development of statistical models in the field of osteoporosis and fragility fractures. Finally, current status and future outlook are discussed and summarized. 


\section{Methods}

A literature search was performed in PubMed using the following keyword: ((("statistical shape") OR ("active shape")) OR (("statistical appearance") OR ("active appearance"))) AND ((osteoporosis) OR (fracture) OR (DXA) OR (BMD) OR (fracture risk) OR (strength) OR (segmentation)). The resulting publications were pre-screened based on the title and abstract to check for the following inclusion criteria:

- Type of publication: original articles

- Type of animals/population: studies dealing with human material

- Language: English

- Publication date: articles published 2015-2021

- Application area: contributions to research within osteoporosis and fragility fractures

\section{Results and Discussion}

The PubMed search was performed on April 16, 2021, and produced 299 results, of which 33 fulfilled the inclusion criteria. Twelve additional papers [11-22] were included, as they fulfilled all inclusion criteria albeit without appearing in the PubMed search. All included papers are listed in Table 1.

\section{Statistical Shape and Appearance Models: Technical Considerations}

Statistical shape models (SSMs) describe the anatomical variation observed in medical images. If a statistical model is built only for the intensity information of the medical images (most commonly bone mineral density, BMD), it is called a statistical appearance model (SAM). Statistical shape and appearance models (SSAMs, Fig. 1) are created when both the shape and the appearance are included into the model. SSM, SAM, and SSAM are usually mathematically based on principal component analysis (PCA). The following subsections describe the most common approaches for statistical shape modeling with emphasis on recent developments.

\section{Shape Representation}

The selected technique for shape representation fundamentally affects the design of the SSM. Point distribution models (PDMs) are the most common class of SSMs. They describe the surface of the modeled object with points, often referred to as landmarks. The points are usually connected to form a mesh. In 2D, the points are set on the contour of the object, and in 3D, the surface of the object is described with a surface mesh. A volume mesh also includes points that are spread inside the specimen and that allow to also describe the internal structure. The point coordinates are collected into one vector $\boldsymbol{x}=\left(x_{1}, y_{1}, z_{1}, \ldots, x_{m}, y_{m}, z_{m}\right)^{T}$ that describes the shape. In other approaches, a parametrized shape representation allows compact but coarse shape description with a few extracted features (e.g., head radius and neck length, for a proximal femur) from the specimen [27].

\section{Shape Refinement}

Recent studies on bone captured the endocortical surface, in addition to periosteal surface. This brings explicit description of the cortical shell and its varying thickness into the shape representation [23, 27]. Typically, the outer or inner cortical surface is first segmented, and then, the cortical thickness is estimated via de-convolution by estimating a point distribution function perpendicular to the bone surface at each surface vertex location. This allows to detect the inner and outer cortical surfaces with sub-pixel accuracy [56].

\section{Appearance Representation}

The most common appearance (sometimes referred to as intensity) in the SAMs is the bone density, which can be expressed, e.g., in Hounsfield units, BMD, or bone volume over total volume (BV/TV). Recently, fabric tensors have also been added into a SSAM together with shape and BV/TV to provide a statistical description of bone anisotropy [16]. Two common approaches exist to describe the appearance:

- Mesh-based: An average volume mesh is deformed on each specimen, and the pixel or voxel intensities covered by each element are captured, e.g., by integrating over each element [25].

- Image-based: Each image is deformed to the shape of an average image, and the density information is captured into the average image's voxels [23].

Please see Bonaretti et al. [57] for the implications of using one approach or the other.

\section{Correspondence Between the Shapes}

Statistical shape and appearance models require that the topology of the node sets is the same; i.e., the anatomical location of each landmark is preserved for all individual shapes. Three common approaches exist to define these point correspondences.

1. Manual identification. An expert sets the points on anatomically distinguishable locations. This approach is most used in 2D problems. 


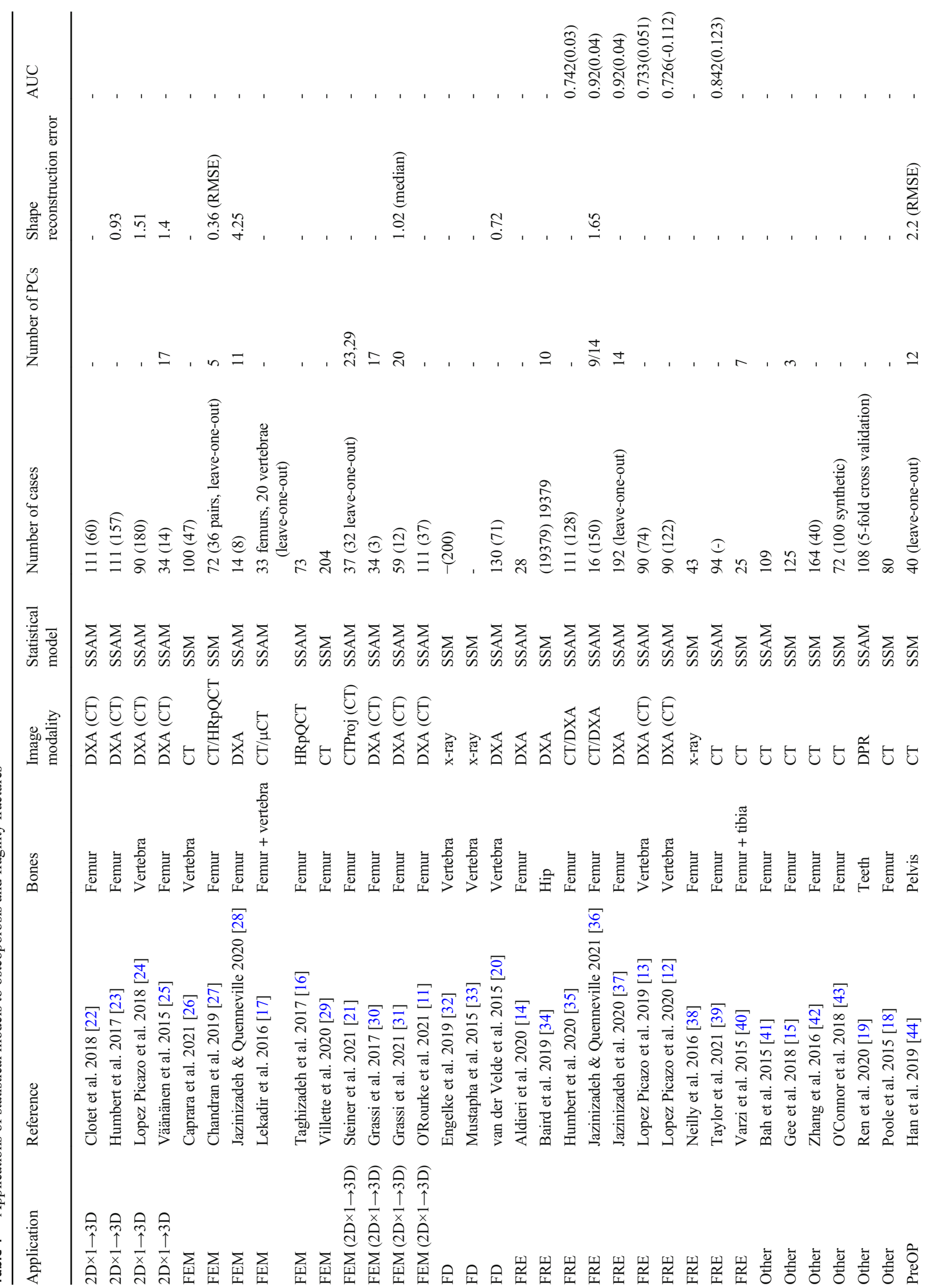




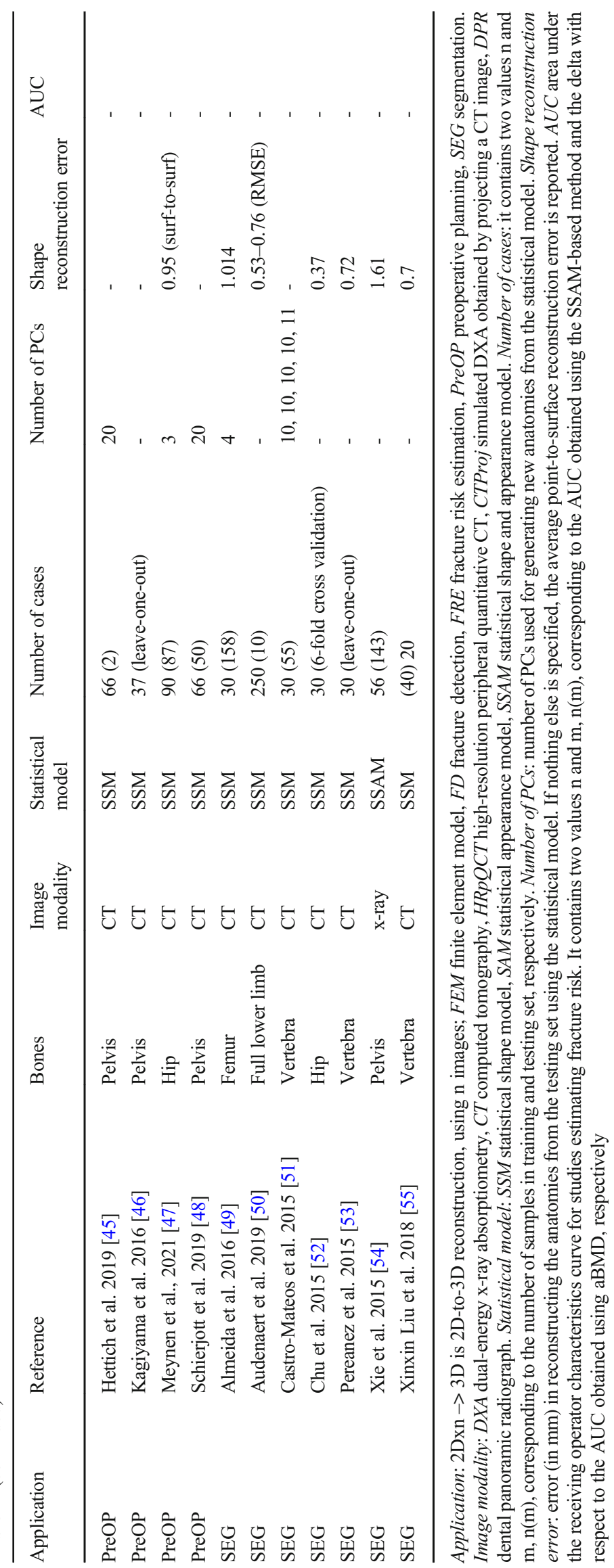


Datasets
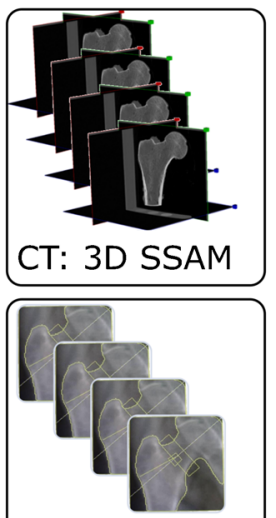

DXA: 2D SSAM

\section{Preprocessing}

1. Segmentation

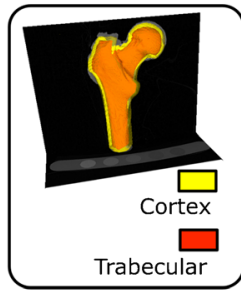

2. Correspondence

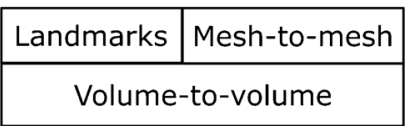

3.Alignment

\begin{tabular}{|l|l|l|} 
Rigid & Similarity & Affine \\
\hline
\end{tabular}
SSAM creation

Parameter reduction with PCA

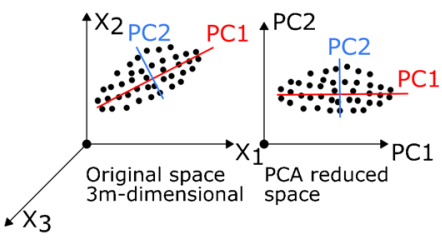

Shape and appearance model $-3 \mathrm{SD}$
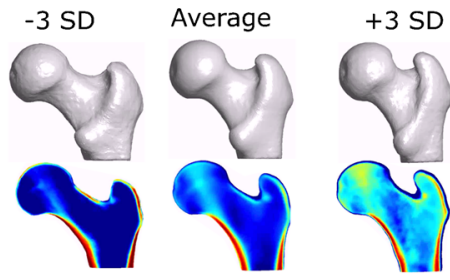

Applications

Pre-operative
planning

Fig. 1 Schematic of the generation of a SSAM and main areas of application. From left to right, a SSAM (or a statistical model of shape or appearance only) can be generated from clinical images, typically CT in case of 3D-based SSAMs and DXA (or X-rays) in case of 2D-based SSAMs. The medical images are segmented to extract the geometries of

2. Mesh-to-mesh correspondence. A reference mesh is registered on each segmented surface mesh, e.g., using B-splines. It has been reported that direct nonlinear mesh registration techniques may cause inaccuracies on anatomical correspondence especially on complex anatomical structures such as the proximal femur [15]. Therefore, techniques have been proposed where mesh registration is done in two steps: first a few anatomically tightly bound landmarks are detected manually or automatically from the target shape, and the reference shape is deformed to match the target shape's landmarks, e.g., using thin-plate splines. Then, the surface mesh is registered more accurately using non-rigid registration techniques such as nonrigid iterative closest point algorithm [15, 25]. Parametrized shape registration is another proposed approach, which generates tight anatomical correspondence [27].

3. Volume-to-volume correspondence. The shape in the reference image is segmented, and the image is registered on each target image.

After the correspondence between the shapes is defined, the latter needs to be aligned. Depending on the problem, the alignment may be rigid, where translations and rotations are removed; e.g., using iterative closest point, it may use similarity alignment where the shapes are also normalized, or it may be affine where also global shear deformations are removed. interest, which are then registered one to another via one of the listed correspondence techniques. Dimensionality reduction is achieved using PCA, thus allowing to build the SSAM. The SSAM can then be applied to many different application areas, as reviewed in the present paper

\section{Dimensionality Reduction}

In PDMs, specimens' vertex coordinates are collected to one observation matrix $\boldsymbol{X}$ with size $2 m \times n$ or $3 m \times n$ depending on the dimensionality of the problem, $m$ is the number of vertices and $n$ number of samples. The deformations between the specimens are assumed linear and normally distributed. The average of each coordinate is calculated as $\widehat{\boldsymbol{X}}=\frac{1}{n} \sum_{i=1}^{n} \boldsymbol{X}_{i}$, and PCA is applied on the sample covariance matrix $\boldsymbol{C}=\frac{1}{n-1} \sum_{i=1}^{n}\left(\boldsymbol{X}_{i}-\widehat{\boldsymbol{X}}\right)\left(\boldsymbol{X}_{i}-\widehat{\boldsymbol{X}}\right)^{T}$. The eigenvectors from PCA, i.e., the principal components (PCs) of shape variation $\boldsymbol{U}_{i}$, can be used to describe the specimen's shape in a compact way as $\boldsymbol{X}_{i} \approx \widehat{\boldsymbol{X}}+\sum_{i=1}^{k} b_{i} \boldsymbol{U}_{i}$, where $b_{i}$ is the principal component score (eigenvalue) of mode $i$.

Many alterations to the classic PDM approach exist. For example, PCA may be calculated directly for observation matrix $\boldsymbol{X}$ using singular value decomposition instead of its covariance matrix $[23,25]$. The PCs can also be calculated iteratively, e.g., with probabilistic PCA [23]. Recently, PDMs have been generalized to Gaussian process morphable models, which allow extending the model beyond the linear span of the observation data and also enable other shape representations than point clouds, such as continuous surface representations [58].

\section{Statistical Shape and Appearance Models}

SSAMs combine in one single statistical model the variation in shape and appearance of a set of medical images. An 
observation matrix $\boldsymbol{X}_{A}$ for the appearance is formed similarly as for the geometry such that each column includes appearance measures for one specimen. Commonly, PCA is calculated separately for geometry and appearance, and these models are compared separately. Alternatively, a third PCA is applied to the combined shape and appearance parameters of two first PCAs allowing analysis of the relation between the shape and appearance. In a more direct approach, the observation matrices of the geometry and appearance can be combined into one matrix $\boldsymbol{X}_{C}=\left[\begin{array}{c}\boldsymbol{X} \\ \boldsymbol{X}_{A}\end{array}\right]$ and thereafter be normalized, e.g., by dividing each row with standard deviation of $\boldsymbol{X}_{C}$ calculated row-wise, allowing one PCA to be applied [25].

\section{Statistical Model Evaluation}

Compactness and generalization error are often calculated for statistical models to evaluate their performance. A compact SSM can represent the shape with fewer PCs. Compactness is calculated as $C(K)=\frac{\sum_{i=1}^{K} \lambda_{i}}{\sum_{j=1}^{N} \lambda_{j}}$, where $K$ is number of the most significant PCs used and $N$ is the number of all PCs. The ratio describes how well the first a few principal components describe the total variation in the data set $[16,47]$. The generalization error is usually calculated with leave-one-out, and it measures how accurately SSAM can generate the specimen dropped out from the model as $G(K)=\frac{1}{N} \sum_{i=1}^{N}\left\|z_{i}(K)-x_{i}\right\|^{2}$, where $z_{i}(K)$ is the shape vector reconstructed with the first $K$ PCs. Another quantitative evaluation metrics is, for example, specificity of a SSAM which evaluates the SSAM's ability to generate realistic new samples [59].

\section{Shape Reconstruction}

One application for SSAMs is generative models where new specimens are sampled based on a training population. One sub-type of this class of application is 2D-to-3D reconstruction where the 3D shape and often internal architecture of a bone is reconstructed based on one or a few 2D radiographs or dual-energy X-ray absorptiometry (DXA) images (see Reyneke et al. [60] for a dedicated methodological review). In these approaches, the 3D SSAM is sampled and digitally reconstructed radiographs (DRR); i.e., projections of the SSAM are generated. The goal is to find the shape parameters and similarity transformation parameters where the DRR best matches the $2 \mathrm{D}$ image. The optimization problem may be solved, e.g., using genetic algorithms [25] or Powell's conjugate directions [23]. For recent developments, also estimation of the cortical thickness has been added into the reconstruction pipeline after the 2D-to-3D reconstruction [24]. However, concerns have been raised whether such technique produces the actual 3D cortical thickness variations or if the extracted cortical thickness mostly reflects general changes in the target image's projected density [61].

\section{Statistical Models Applied in Studies of Osteoporosis and Fragility Fractures}

In this section, the most recent applications of SSAMs related to osteoporosis and fracture risk assessment are reviewed and summarized. The section is divided by applications areas (Table 1): image segmentation, preoperative planning, 2Dto-3D reconstruction, finite element (FE) analysis, fracture risk estimation, fracture detection, and finally other applications.

\section{Segmentation}

One of the more traditional applications of statistical models in osteoporosis and medical imaging is to enable automatic or semi-automatic segmentation of medical images. Automatic segmentation is also often the first step in many of the following approaches, e.g., fracture detection, subject-specific numerical modeling, and preoperative planning. The challenge in the segmentation process is typically to initialize the process. Thereafter, fitting a SSAM (or SSM or SAM) to a target is automatic per se.

All recent applications of SSAMs to automatic segmentation were either targeted to vertebrae $[51,53,55]$ or to hip or full lower limb [49, 50, 52, 54]. Interestingly, all studies except one [54] focused on segmentation of computed tomography (CT) images, despite CT not being commonly adopted in clinical care of osteoporosis. All methods are automatic or semi-automatic, where human interaction typically consists in manually picking some anatomical landmarks on the target image [54]. These landmarks are then used for a first rough alignment of the SSAM. This manual step can be avoided by, e.g., using an automatic rough segmentation based on automatic thresholding for the same purpose [50]. All studies reported reconstruction errors below $1 \mathrm{~mm}$ in terms of average point-to-surface distance, showing that SSAM-based segmentation of CT images can be considered a well-established field. An interesting new application consists in the use of articulated SSAMs [50-52]. Essentially, these are models consisting of SSAMs of several adjacent bodies, where the interspace between the adjacent objects is also modeled and controlled to avoid overlaps and intersections between the different bodies. This technique can be useful to model large anatomical segments $[50,51]$ or to deal with overlapping features in the CT images, for example, at the hip joint [52]. When high segmentation accuracies are required, the opposite approach can be followed, i.e., using statistical decomposition to build the SSAM of one single body. Improvement of 16-19\% in reconstruction accuracy was reported for human vertebrae using this approach [53]. 


\section{Preoperative Planning}

One of the most popular applications of SSAM-based segmentation is to use it for preoperative planning, i.e., to plan the optimal position of an implant given the subject-specific anatomy of the patient. All recent studies in this direction focused on the hip, which is not surprising given the importance and frequency of total hip arthroplasty (THA) due to both osteoporosis and osteoarthritis.

Most studies [45, 47, 48] are based on generating a SSAM from healthy pelvic anatomies and then fit the SSAM to the patient's anatomy to quantify the severity of the acetabular defects, thus assisting in preoperative planning. Common to these studies is the limitation that they require manual segmentation of the target pelvis as well as identification/ labeling of the pathological areas. This makes it timeconsuming and not widely implementable in clinical practice. Given the promising results of the SSAM-based segmentation algorithms shown in the previous section, this calls for collaboration between research groups that focus on the different applications, as well as for further development of the segmentation algorithms to increase robustness for pathological or defect anatomies. Kagiyama et al. [46] proposed an automatic method to assess the acetabular cup placement, based on a combined pelvis-cup merged SSM. The latter is registered to the patient anatomy by only accounting for the pelvic part of the SSM, thus "dragging along" the cup to predict its optimal placement. The method showed inferior results when compared to manual segmentation and cup placement, especially for cases where the size of the defect was larger. A common problem with studies proposing automatic preoperative planning of THA is that an established gold standard does not seem to exist. Moreover, the long-term benefits of a potentially improved implant placement are hard to quantify due to the need of large clinical trials that are expensive and not justified by the results obtained to date. Han et al. [44] instead proposed a SSM of the healthy pelvis that also included an atlas of possible trajectories for K-wire insertions, aimed at being registered over fluoroscopic images to augment them with the insertion trajectory for better planning. So far, this method was tested only with leave-one-out in the SSM training set, the latter consisting of non-fractured pelvises from the Cancer Imaging Archive. Further validation using true preoperative CT images is advocated to show clinical feasibility.

\section{D-to-3D Reconstruction}

Another application of SSAMs is 3D reconstruction of shape and appearance from clinical 2D images. The idea is to find the $3 \mathrm{D}$ object whose $2 \mathrm{D}$ projection would look like the target image. This mathematical problem has an infinite number of solutions, but by using the information about anatomical variability contained in the SSAM, a clinically relevant solution can be obtained.

Two main approaches for 2D-to-3D reconstruction from DXA images were found in recent literature. Väänänen et al. [25] used mesh-based SSAMs of proximal femur and pelvis to reconstruct $3 \mathrm{D}$ femoral anatomies from DXA images, where the pelvis was used to account for its shadowing. The 2D-to$3 \mathrm{D}$ reconstruction was based on optimizing a cost function that included sum of absolute differences between the target DXA and the digitally reconstructed radiograph of the SSAM instance. The method provided average reconstruction errors of $1.4 \mathrm{~mm}$ for the shape and $0.2 \mathrm{~g} / \mathrm{cm}^{3}$ for the volumetric BMD (vBMD) when evaluated on clinical DXA scans. Validation on different types of DXA images evidenced how signal-to-noise ratio, and not image resolution, is the key parameter to obtain an accurate reconstruction.

Humbert et al. [23] proposed an approach for 2D-to-3D reconstruction that uses an image-based SSAM, producing a so-called 3D DXA as output. The latter can be processed like a conventional CT scan to segment its shape, generate an FE model, or calculate morphological parameters. Reconstruction errors of $0.93 \mathrm{~mm}$ for the shape and $0.72 \mathrm{~g} / \mathrm{cm}^{3}$ for the cortical vBMD were reported. Crucially, the method is able to provide a solution within minutes, compared to many hours required for the approach by Väänänen et al. [25]. Due to its speed and its commercial availability, the method has been increasingly adopted by other research groups, as shown in the following sections. The method has been used both on proximal femurs [22] and vertebrae [24]. In the first case, the accuracy of hip structural parameters calculated on the reconstructed 3D DXA was evaluated, showing an overall good accuracy, but also non-reliable structural measurements at the pelvis and femoral head. This is likely because no SSAM of the pelvis is used; thus, the reconstruction cannot account for its shadowing, and the femoral head is not fitted.

\section{Finite Element Analysis}

One of the applications of SSAMs that is increasingly popular is FE analysis. SSAM-based FE models could be aimed at, e.g., predicting subject-specific bone strength from clinical images $[21,28,30,31]$, evaluating the efficacy of treatment [11], investigating the effect of femoral anatomy on its strength [29], estimating range of motions [26], and providing augmented information to clinical images [16, 17].

Subject-specific prediction of bone strength using FE models has been suggested as a way to improve fracture risk prediction for over 20 years [62]. Most subject-specific FE modeling approaches rely on the availability of 3D CT images. However, their availability is scarce for clinical assessment of fracture risk, where 2D DXA is routinely used, instead. SSAM-based FE models can overcome this by producing FE models directly and automatically from DXA scans. 
Grassi et al. used the 2D-to-3D reconstruction methodology from Väänänen et al. [25] to generate 3D subject-specific FE models from 2D DXA scans and predict femoral strength. Crucially, the predictions were validated against gold standard CT-based FE models as well as ex vivo experimental measurement. Accurate results were shown for both quasi-static FE simulations of single-leg-stance $(\mathrm{SEE}=1215 \mathrm{~N}$ over 3 bones with 3 different DXA images each [30]) and biofidelic dynamic simulations of a fall to the side (correct prediction of the fall outcome in terms of fracture/non-fracture in 11 out of 12 cases [31], same as gold standard CT-based models [63]).

Recently, Steiner et al. [21] developed a SSAM together with a $2 \mathrm{D}-$ to-3D reconstruction that works with either a single or two orthogonal DXA images. The FE predictions of the 3D reconstructed FE models were validated for 32 femurs, making this the largest validation study for 2D-to-3D reconstructed FE models. However, the reconstruction accuracy for shape and BMD was inferior to other studies (2.2 mm using one simulated DXA image versus $1.0 \mathrm{~mm}$ from Väänänen et al. [25]). FE predictions largely underestimated femoral strength when compared to experimental measurements $(y=0.39 \mathrm{x}+707 \mathrm{~N}$ for the $2 \mathrm{D}$-to-3D reconstructed FE models from 1 single simulated DXA, based on digitized data from fig 12 , [21]), albeit with an acceptable correlation $\left(R^{2}=0.72\right.$, $\mathrm{SEE}=1870 \mathrm{~N}$ ). The method performed better when two orthogonal DXA scans were used, which are however not commonly available in clinical practice.

Another approach to obtain subject-specific FE predictions of individual bone strength from DXA images consists in building 2D subject-specific FE models, thus avoiding the need for 2D-to-3D reconstruction. To this purpose, Jazinizadeh and Quenneville [28] developed a 2D SSAM of the proximal femur. Linear regression between predicted fracture load and experiments showed an average coefficient of determination of 0.68 , with a range between 0.55 and 0.82 depending on which specimens were selected to be included in the training set and which in the test set.

SSAM-based FE models can be used to evaluate the effect or preventive treatment. For example, O'Rourke et al. [11] used the 2D-to-3D reconstruction method by Humbert et al. [23] and built FE models from the reconstructed 3D DXA to predict femoral strength in a cohort of men where DXA images were acquired pre- and post-exercise interventions. Additionally, femoral strength was predicted for a cohort of women with same-day repeated DXA scans (where 0\% strength change should be expected). However, differences up to $62 \%$ for predicted strength were reported between two same-day repeated DXA scans, showing that the method is not reliable on a subject-specific basis.

Another classic application of SSAM-based FE models is to create synthetic anatomies from the SSAM with the aim to investigate the effect of anatomical features on femoral strength and fracture risk. Villette et al. [29] used the SSM methodology by Zhang et al. [64] to generate 7 synthetical anatomies representing the average femur and variations of \pm 2 SD for the most influential PCs. Results suggest that variations of femoral shapes, especially different neck-shaft angles, can affect the strength of the femur. However, the low sample size makes it difficult to generalize the findings.

A novel application of SSAM-based FE models is the augmentation of information that can be extracted from clinical CT images. The basic idea is to combine a SSAM from clinical CT and a SAM of anisotropy information from $\mu \mathrm{CT}$ or high-resolution peripheral quantitative CT (HR-pQCT). Then, a statistical predictive model can be used to find relationships between the statistical models, with the goal to identify if and how anisotropy information can be inferred from bone shape or BMD distribution. The SSAM-based augmented FE models predicted strength of femurs and vertebrae with an average error of $2 \%$ and $4 \%$, respectively, when compared to gold standard micro-CT-based FE predictions [17]. However, another study reported a very low compactness of the SAM of the fabric tensor, indicating that the SAM of the fabric tensor is not able to complement the FE analysis much over the average distribution of fabric tensor. Consistently, no correlation was found between the distributions of bone shape, $\mathrm{BV} / \mathrm{TV}$, and fabric tensor [16]. Thus, it may not be possible to gain relevant information on the degree of anisotropy from only the shape or density of a patient, which are those available from clinical resolution CT images.

Another trend that can be observed, thanks to the increasing computational power, is to adopt SSAMs to perform only small parts of an FE modeling pipeline. For example, PCs can be further used for supervised learning aimed at obtaining shape regression [27], or the SSM can be fitted to an automatic segmentation to obtain isotopological meshes for all samples, with benefits of, e.g., applying consistent boundary conditions [26].

\section{Fracture Risk Estimation}

Many recent applications of SSAMs are aimed at predicting fracture risk in subjects affected by osteoporosis. Most of the applications use the PC scores to look for statistically significant differences between them in fracture versus controls cases, or feed the PC scores as input variables in logistic regression analyses [14, 36-38, 40]. Other interesting approaches aimed at finding associations between PC scores and genetic polymorphisms that in turn are known to influence fracture risk [34], or at using 2D-to-3D reconstruction to use vBMD to predict fracture risk [12, 13, 35]. Only one study was found that used the strength calculated by SSAM-based FE models to predict fracture risk [39].

Most studies used 2D SSM or SSAM to predict fracture risk $[14,34,36,37,40]$ in an effort to make the method fast and compatible with clinically available DXA images. 
However, the improvements in area under the receiver operating characteristic (aROC) curve were not statistically significant when compared to aBMD alone [36, 37]. Aldieri et al. [14] proposed to use partial least squares (PLS) instead of PCA for dimensionality reduction. In PLS, an additional response variable $\mathrm{Y}$ can be accounted for, in this case the fracture risk, so that the covariance between the input matrix $\mathrm{X}$ and $\mathrm{Y}$ is maximized. This allows to find attributes that are relevant to fracture risk, rather than only those with maximum covariance. However, the study only compared the findings to a surrogate for fracture risk (femoral strength predicted by CTbased FE models). Interestingly, Jazinizadeh and Quenneville [36] compared the performance of 2D-based and 3D-based SSAM (the latter combined with a 2D-to-3D reconstruction) for predicting fracture risk, showing no statistically significant differences between the two in terms of area under the ROC curve. This suggest that, when using PC scores for logistic regression purposes, 2D SSAMs are better to estimate fracture risk, given their simplicity and lower computational cost. Other approaches, however, used 3D SSAMs and 2D-to-3D reconstruction to produce measurements of vBMD for cortical and trabecular compartment, using those as predictors of fracture risk instead of aBMD from DXA images [12, 13, 35]. Also here, small but not statistically significant improvements in terms of area under the ROC curve were predicted when compared to aBMD.

Taylor et al. [39] recently raised the question of whether we are reaching the limits of information that can be extracted from an image to predict the risk of fracture. To answer this question, SSAM-based FE models were built for 94 subjects in a case-control cohort, and the femoral strength was predicted. Logistic regression classification models were used to predict fracture risk, using only the PCs that were predictive of femoral strength. Results show that most of the PCs do not contribute to femoral neck strength and were hence unlikely to improve the prediction of fracture risk. Consequently, the authors conclude that we are indeed approaching the limit of what can be achieved by an image alone.

A limitation with all studies is that they are based on retrospective case-control cohorts of subjects, in some cases with peculiar choices for subjects' selection. Also, most studies only use the PC scores to build logistic regression classification models, and no study has yet used bone strength calculated from SSAM-based 2D-to-3D reconstruction. Thus, it is necessary that further studies corroborate the validity of SSAM-based FE models by providing additional experimental validation, as detailed in the previous section. That, however, leaves hope that there is more to exploit from a clinical image to improve fracture risk estimation.

\section{Fracture Detection}

Another useful area of application of statistical models is vertebral fracture detection. Some vertebral fractures are asymptomatic, yet they are predictive of future major osteoporotic fractures. However, many vertebral fractures can be overlooked even on X-ray or DXA images. Statistical models can provide a more quantitative assessment based on automatic or semi-automatic image segmentation and anatomical landmark identification [20,32,33]. All studies consistently reported that the method works best for mild fractures $(<25 \%$ loss of height, [41]), which are also the most difficult to detect. This compensates for the issues in robustness (manual correction needed in $15 \%$ of the cases [32]) occurring especially for more severe fractures, for which SSMs built from healthy anatomies cannot reach a good fit.

\section{Other Applications}

Some relevant applications of statistical models could not fit any of the categories above $[15,18,19,42,43,65]$. Three of these studies $[15,42,65]$ focused on anatomical measurements and assessment of cortical thickness, all parameters known to be relevant contributors to femoral strength and fracture risk. O'Connor et al. [43] generated 100 synthetic anatomies using a SSM to span the whole range of anatomical variability, with the aim of assessing the effect of combined flexion and external rotation on anatomical measurements of the proximal femur from $2 \mathrm{D}$ radiographic images. Not surprisingly, the study showed that such rotations, and in particular the combination of them, can significantly affect the quality of the anatomical measurements from radiographic images. Using SSAMs and 2D-to-3D reconstruction could actually help mitigate such issues [66].

Ren et al. [9] used 2D SSM and SAM to automatically detect anatomical landmarks in dental panoramic radiograph images. These landmarks are needed to perform osteoporosis pre-screening.

Poole et al. [18] used statistical parametric mapping and a procedure similar to Gee et al. [15] to determine the effects of denosumab treatment on cortical thickness and density. After 36 months, most of the femoral cortex of denosumab-treated patients showed a statistically significant increase in cortical mass surface density (i.e., cortical thickness * cortical density), but that some critical locations for femoral strength, such as the lateral trochanter, already showed significant increase after 12 months. This can explain the efficacy of denosumab treatment from a biomechanical perspective. 


\section{Conclusions}

This review aimed at summarizing recent advancements in development and use of statistical models of shape and appearance in areas connected to osteoporosis and fragility fractures. SSAM-based models allow to generate subject-specific numerical models in an automatic and clinically feasible fashion, ultimately providing quantitative information that could assist in clinical decision-making. Recent applications have also proposed SSAMs as a tool to provide augmented information from medical images.

A comparison with a similar review study published in 2014 [10] highlights a decreasing number of studies about SSAM-based image segmentation, while some implementations begin to be available inside commercial software [67]. These are signs that the techniques for SSAMbased image segmentation have reached a relatively mature stage. On the contrary, many more studies have taken the important step to test SSAM-based methods for estimating fracture risk in clinical cohorts during the past five years. The application area of fracture risk estimation is in a sense emblematic of the status of SSAM-based approaches in osteoporosis. On the one hand, a raising number of studies are published on the topic, proposing applications that are constantly getting closer to clinical applications, with some tools being commercialized and certified as medical devices [23]. On the other hand, we may be approaching the limit of what can be extracted from an image alone, as suggested in one of the studies [39]. While this may give the impression that there is little space for further improvement in the near future, we still see substantial room for improvements in many areas. Most importantly, we call for openly accessible benchmarks that would help to compare the performance of the different approaches more objectively. We could not find two studies from different research groups validating their methods on the same data set. Instead, we found some peculiar choices when it comes to validation sets, especially when case-control studies from clinical cohorts are performed, whose effects can possibly overshadow those of the actual SSAM-based techniques. The need of openly accessible and widely used benchmarks is not exclusive to SSAMs, and there is hope that the recent attention to data sharing from both scientific community and funding agencies may spark incentives for the near future. Secondly, benchmarks should be used to validate every step of the proposed SSAM-based techniques in a consequential manner. For example, a study implementing SSAM-based 2D-to-3D reconstruction to produce subject-specific FE models should first validate the reconstruction ability of the $2 \mathrm{D}-$ to-3D reconstruction and then validate the predictive ability of the generated FE models against experimental measurements. A thorough, stepwise validation of SSAM-based methods against openly accessible and widely accepted benchmarks could provide the needed credibility to bring these methods closer to the daily clinical practice of osteoporosis.

Funding Open access funding provided by Lund University.

Open Access This article is licensed under a Creative Commons Attribution 4.0 International License, which permits use, sharing, adaptation, distribution and reproduction in any medium or format, as long as you give appropriate credit to the original author(s) and the source, provide a link to the Creative Commons licence, and indicate if changes were made. The images or other third party material in this article are included in the article's Creative Commons licence, unless indicated otherwise in a credit line to the material. If material is not included in the article's Creative Commons licence and your intended use is not permitted by statutory regulation or exceeds the permitted use, you will need to obtain permission directly from the copyright holder. To view a copy of this licence, visit http://creativecommons.org/licenses/by/4.0/.

\section{References}

Papers of particular interest, published recently, have been highlighted as:

- Of importance

-• Of major importance

1. Borgström F, Karlsson L, Ortsäter G, Norton N, Halbout P, Cooper $\mathrm{C}$, et al. Fragility fractures in Europe: burden, management and opportunities. Arch Osteoporos. 2020 Apr 19;15(1):59.

2. Odén A, McCloskey EV, Kanis JA, Harvey NC, Johansson H. Burden of high fracture probability worldwide: secular increases 2010-2040. Osteoporos Int. 2015 Sep 1;26(9):2243-8.

3. Stone KL, Seeley DG, Lui L-Y, Cauley JA, Ensrud K, Browner WS, Nevitt MC, Cummings SR, Osteoporotic Fractures Research Group. BMD at multiple sites and risk of fracture of multiple types: long-term results from the study of osteoporotic fractures. J Bone Miner Res. 2003 Nov 1;18(11):1947-54.

4. Wainwright SA, Marshall LM, Ensrud KE, Cauley JA, Black DM, Hillier TA, Hochberg MC, Vogt MT, Orwoll ES, Study of Osteoporotic Fractures Research Group. Hip fracture in women without osteoporosis. J Clin Endocrinol Metab. 2005 May 1;90(5):2787-93.

5. Dyer SM, Crotty M, Fairhall N, Magaziner J, Beaupre LA, Cameron ID, et al. A critical review of the long-term disability outcomes following hip fracture. BMC Geriatr. 2016 Sep 2;16(1): 158.

6. Cootes T, Taylor C. Statistical models of appearance for medical image analysis and computer vision. Med Imaging. 2001;2001: 236-48.

7. Cootes TF, Taylor CJ, Cooper DH, Graham J. Active shape models-their training and application. Comput Vis Image Underst. 1995 Jan 11;61(1):38-59.

8. Cootes TF, Taylor CJ, Cooper DH, Graham J. Training models of shape from sets of examples. In: Hogg DC, Boyle RD, editors. Training. Springer-Verlag; 1992. p. 9-18.

9. Heimann T, Meinzer H-P. Statistical shape models for 3D medical image segmentation: a review. Med Image Anal. 2009 Aug;13(4): 543-63. 
10. Castro-Mateos I, Pozo JM, Cootes TF, Wilkinson JM, Eastell R, Frangi AF. Statistical shape and appearance models in osteoporosis. Curr Osteoporos Rep. 2014 Jun 2;12(2):163-73.

11. O'Rourke D, Beck BR, Harding AT, Watson SL, Pivonka P, Martelli S. Assessment of femoral neck strength and bone mineral density changes following exercise using 3D-DXA images. J Biomech. 2021 Apr 15;119:110315.

12. López Picazo M, Humbert L, Winzenrieth R, Di Gregorio S, González Ballester MA, del Río Barquero LM. Association between osteoporotic femoral neck fractures and DXA-derived 3D measurements at lumbar spine: a case-control study. Arch Osteoporos. 2020 Jan 3;15(1):8.

13. López Picazo M, Humbert L, Di Gregorio S, González Ballester MA, del Río Barquero LM. Discrimination of osteoporosis-related vertebral fractures by DXA-derived 3D measurements: a retrospective case-control study. Osteoporos Int. 2019 May 1;30(5):1099110 .

14. Aldieri A, Terzini M, Audenino AL, Bignardi C, Morbiducci U. Combining shape and intensity dxa-based statistical approaches for osteoporotic HIP fracture risk assessment. Comput Biol Med. 2020 Dec 1;127:104093.

15.• Gee AH, Treece GM, Poole KES. How does the femoral cortex depend on bone shape? A methodology for the joint analysis of surface texture and shape. Med Image Anal. 2018 Apr;45:55-67. A well written manuscript that indulges on many small important, yet often overlooked, technical details about building an SSM. Highly useful if you are planning to build your own statistical mode.

16. Taghizadeh E, Chandran V, Reyes M, Zysset P, Büchler P. Statistical analysis of the inter-individual variations of the bone shape, volume fraction and fabric and their correlations in the proximal femur. Bone. 2017;103:252-61. This manuscript proposes one of the novelties presented during the last years, i.e., the inclusion of a second appearance parameter in a SSAM. It features a clear and honest discussion of the results.

17. Lekadir K, Noble C, Hazrati-Marangalou J, Hoogendoorn C, van Rietbergen B, Taylor ZA, Frangi AF. Patient-specific biomechanical modeling of bone strength using statistically-derived fabric tensors. Ann Biomed Eng. 2016;44:234-46.

18. Poole KE, Treece GM, Gee AH, Brown JP, McClung MR, Wang $\mathrm{A}$, et al. Denosumab rapidly increases cortical bone in key locations of the femur: a 3D bone mapping study in women with osteoporosis. J Bone Miner Res. 2015;30(1):46-54.

19. Ren J, Fan H, Yang J, Ling H. Detection of trabecular landmarks for osteoporosis prescreening in dental panoramic radiographs. In: 2020 42nd Annual International Conference of the IEEE Engineering in Medicine Biology Society (EMBC). 2020. p. 2194-7.

20. van der Velde R, Ozanian T, Dumitrescu B, Haslam J, Staal J, Brett A, van den Bergh J, Geusens P. Performance of statistical models of shape and appearance for semiautomatic segmentations of spinal vertebrae T4-L4 on digitized vertebral fracture assessment images. Spine J. 2015 Jun 1;15(6):1248-54.

21. Steiner L, Synek A, Pahr DH. Femoral strength can be predicted from $2 \mathrm{D}$ projections using a 3D statistical deformation and texture model with finite element analysis. Med Eng Phys. 2021 May 24;93:72-82.

22. Clotet J, Martelli Y, Di Gregorio S, del Río Barquero LM, Humbert L. Structural parameters of the proximal femur by 3-dimensional dual-energy X-ray absorptiometry software: comparison with quantitative computed tomography. J Clin Densitom . 2018;21:550-62.

23. Humbert L, Martelli Y, Fonollà R, Steghöfer M, Di Gregorio S, Malouf J, et al. 3D-DXA: assessing the femoral shape, the trabecular macrostructure and the cortex in 3D from DXA images. IEEE Trans Med Imaging. 2017 Jan;36(1):27-39.
24. López Picazo M, Magallón Baro A, Del Río Barquero LM, Di Gregorio S, Martelli Y, Romera J, et al. 3-D subject-specific shape and density estimation of the lumbar spine from a single anteroposterior DXA image including assessment of cortical and trabecular bone. IEEE Trans Med Imaging. 2018 Dec;37(12): 2651-62.

25. Väänänen SP, Grassi L, Flivik G, Jurvelin JS, Isaksson H. Generation of 3D shape, density, cortical thickness and finite element mesh of proximal femur from a DXA image. Med Image Anal. 2015;24(1):125-34.

26. Caprara S, Carrillo F, Snedeker JG, Farshad M, Senteler M. Automated pipeline to generate anatomically accurate patientspecific biomechanical models of healthy and pathological FSUs. Front Bioeng Biotechnol. 2021;9:636953.

27. Chandran V, Maquer G, Gerig T, Zysset P, Reyes M. Supervised learning for bone shape and cortical thickness estimation from CT images for finite element analysis. Med Image Anal. 2019 Feb 1;52: $42-55$.

28. Jazinizadeh F, Quenneville CE. Enhancing hip fracture risk prediction by statistical modeling and texture analysis on DXA images. Med Eng Phys. 2020 Apr 1;78:14-20.

29. Villette CC, Zhang J, Phillips ATM. Influence of femoral external shape on internal architecture and fracture risk. Biomech Model Mechanobiol. 2020 Aug 1;19(4):1251-61.

30. Grassi L, Väänänen SP, Ristinmaa M, Jurvelin JS, Isaksson H. Prediction of femoral strength using 3D finite element models reconstructed from DXA images: validation against experiments. Biomech Model Mechanobiol. 2017 Dec 21;16(3):1-12.

31. Grassi L, Fleps I, Sahlstedt H, Väänänen SP, Ferguson SJ, Isaksson $\mathrm{H}$, Helgason B. Validation of 3D finite element models from simulated DXA images for biofidelic simulations of sideways fall impact to the hip. Bone. 2021 Jan 1;142:115678.

32. Engelke K, Stampa B, Steiger P, Fuerst T, Genant HK. Automated quantitative morphometry of vertebral heights on spinal radiographs: comparison of a clinical workflow tool with standard 6point morphometry. Arch Osteoporos. 2019 Feb 11;14(1):18.

33. Mustapha A, Hussain A, Samad SA, Zulkifley MA, Diyana Wan Zaki WM, Hamid HA. Design and development of a content-based medical image retrieval system for spine vertebrae irregularity. Biomed Eng Online. 2015 Jan 16;14(1):6.

34. Baird DA, Evans DS, Kamanu FK, Gregory JS, Saunders FR, Giuraniuc CV, Barr RJ, Aspden RM, Jenkins D, Kiel DP, Orwoll ES, Cummings SR, Lane NE, Mullin BH, Williams FMK, Richards JB, Wilson SG, Spector TD, Faber BG, et al. Identification of novel loci associated with hip shape: a meta-analysis of genome wide association studies. J Bone Miner Res. 2019;34(2):241-51.

35.• Humbert L, Bagué A, Di Gregorio S, Winzenrieth R, Sevillano X, González Ballester MÁ, et al. DXA-based 3D analysis of the cortical and trabecular bone of hip fracture postmenopausal women: a case-control study. J Clin Densitom. 2020 Jul 1;23(3):40310. This is one the latest papers from the group that so far is the only one able to commercialize their method for 2D-to-3D reconstruction of DXA images using a SSAM-based approach.

36. Jazinizadeh F, Quenneville CE. 3D Analysis of the Proximal Femur Compared to 2D Analysis for Hip Fracture Risk Prediction in a Clinical Population. Ann Biomed Eng. 2021 Apr 1;49(4):1222-32. This is one of the few studies that compares the performance of 2D-based versus 3D-based SSAMs when used to predict fracture risk from clinical DXA images.

37. Jazinizadeh F, Adachi JD, Quenneville CE. Advanced 2D image processing technique to predict hip fracture risk in an older population based on single DXA scans. Osteoporos Int. 2020 Oct 1;31(10):1925-33.

38. Neilly D, Khan SK, Gregory JS, Aspden RM, Hutchison JD, Deehan DJ. Can radiographs of hip fractures predict subsequent 
hip fractures? A shape modelling analysis. Injury. 2016 Jul 1;47(7): 1543-6.

39. Taylor M, Viceconti M, Bhattacharya P, Li X. Finite element analysis informed variable selection for femoral fracture risk prediction. J Mech Behav Biomed Mater. 2021 Mar 4;118:104434. This is an interesting study aimed at assessing fracture risk using an SSAM-based approach. The research question is broad, as the authors try to assess whether we are reaching the limits of the information that can be extracted from a clinical image.

40. Varzi D, Coupaud SAF, Purcell M, Allan DB, Gregory JS, Barr RJ. Bone morphology of the femur and tibia captured by statistical shape modelling predicts rapid bone loss in acute spinal cord injury patients. Bone. 2015 Dec 1;81:495-501.

41. Genant HK, Wu CY, van Kuijk C, Nevitt MC. Vertebral fracture assessment using a semiquantitative technique. J Bone Miner Res. 1993;8(9):1137-48.

42. Zhang J, Hislop-Jambrich J, Besier TF. Predictive statistical models of baseline variations in 3-D femoral cortex morphology. Med Eng Phys. 2016 Mar;38(5):450-7.

43. O'Connor JD, Rutherford M, Hill JC, Beverland DE, Dunne NJ, Lennon $\mathrm{AB}$. Effect of combined flexion and external rotation on measurements of the proximal femur from anteroposterior pelvic radiographs. Orthop Traumatol Surg Res. 2018 Jun 1;104(4):44954.

44. Han R, Uneri A, Silva TD, Ketcha M, Goerres J, Vogt S, et al. Atlas-based automatic planning and 3D-2D fluoroscopic guidance in pelvic trauma surgery. Phys Med Biol. 2019 May;64(9):095022.

45. Hettich G, Schierjott RA, Ramm H, Graichen H, Jansson V, Rudert M, Traina F, Grupp TM. Method for quantitative assessment of acetabular bone defects. J Orthop Res. 2019;37(1):181-9.

46. Kagiyama Y, Otomaru I, Takao M, Sugano N, Nakamoto M, Yokota F, Tomiyama N, Tada Y, Sato Y. CT-based automated planning of acetabular cup for total hip arthroplasty (THA) based on hybrid use of two statistical atlases. Int J CARS. 2016 Dec 1;11(12):2253-71.

47. Meynen A, Vles G, Zadpoor AA, Mulier M, Scheys L. The morphological variation of acetabular defects in revision total hip arthroplasty - a statistical shape modeling approach. J Orthop Res. 2021.

48. Schierjott RA, Hettich G, Graichen H, Jansson V, Rudert M, Traina F, Weber P, Grupp TM. Quantitative assessment of acetabular bone defects: a study of 50 computed tomography data sets. PLoS One. 2019 Oct 17;14(10):e0222511.

49. Almeida DF, Ruben RB, Folgado J, Fernandes PR, Audenaert E, Verhegghe B, de Beule M. Fully automatic segmentation of femurs with medullary canal definition in high and in low resolution CT scans. Med Eng Phys. 2016 Dec 1;38(12):1474-80.

50. Audenaert EA, Houcke JV, Almeida DF, Paelinck L, Peiffer M, Steenackers G, et al. Cascaded statistical shape model based segmentation of the full lower limb in CT. Comput Methods Biomech Biomed Eng. 2019 Apr 26;22(6):644-57. This manuscript presents a method to segment the full lower limb from CT images using an SSM-based method. It clearly explains the methods and presents interesting results.

51. Castro-Mateos I, Pozo JM, Pereañez M, Lekadir K, Lazary A, Frangi AF. Statistical interspace models (SIMs): application to robust 3D spine segmentation. IEEE Trans Med Imaging. 2015 Aug;34(8):1663-75.

52. Chu C, Chen C, Liu L, Zheng G. FACTS: fully automatic ct segmentation of a hip joint. Ann Biomed Eng. 2015;43(5):1247-59.

53. Pereañez M, Lekadir K, Castro-Mateos I, Pozo JM, Lazáry Á, Frangi AF. Accurate segmentation of vertebral bodies and processes using statistical shape decomposition and conditional models. IEEE Trans Med Imaging. 2015 Aug;34(8):1627-39.

54. Xie W, Franke J, Chen C, Grützner PA, Schumann S, Nolte L-P, Zheng G. A complete-pelvis segmentation framework for imagefree total hip arthroplasty (THA): methodology and clinical study. Int J Med Robot Comput Assist Surg. 2015;11(2):166-80.

55. Liu X, Yang J, Song S, Cong W, Jiao P, Song H, Ai D, Jiang Y, Wang Y. Sparse intervertebral fence composition for 3D cervical vertebra segmentation. Phys Med Biol. 2018 Jun;63(11):115010.

56. Treece GM, Gee AH. Independent measurement of femoral cortical thickness and cortical bone density using clinical CT. Med Image Anal. 2015;20(1):249-64.

57. Bonaretti S, Seiler C, Boichon C, Reyes M, Buchler P. Image-based vs. mesh-based statistical appearance models of the human femur: implications for finite element simulations. Med Eng Phys. 2014;36(12):1626-35.

58. Lüthi M, Gerig T, Jud C, Vetter T. Gaussian process morphable models. IEEE Trans Pattern Anal Mach Intell. 2018 Aug;40(8): 1860-73.

59. Goparaju A, Bone A, Hu N, Henninger HB, Anderson AE, Durrleman S, et al. Benchmarking off-the-shelf statistical shape modeling tools in clinical applications. arXiv:200902878. 2020 Sep 6.

60. Reyneke CJF, Lüthi M, Burdin V, Douglas TS, Vetter T, Mutsvangwa TEM. Review of 2-D/3-D reconstruction using statistical shape and intensity models and X-ray image synthesis: toward a unified framework. IEEE Rev Biomed Eng. 2019;12: 269-86. This manuscript includes a dedicated review on the topic of 2D-to-3D reconstruction. Useful to get a deep insight into the technical aspects of it.

61. Whitmarsh T. Concerns regarding the use of 3D-DXA. Bone. 2021 Mar;27:115939.

62. Cody DD, Gross GJ, Hou FJ, Spencer HJ, Goldstein SA, Fyhrie DP. Femoral strength is better predicted by finite element models than QCT and DXA. J Biomech. 1999;32(10):1013-20.

63. Fleps I, Guy P, Ferguson SJ, Cripton PA, Helgason B. Explicit finite element models accurately predict subject-specific and velocity-dependent kinetics of sideways fall impact. J Bone Miner Res. 2019;34:1837-50.

64. Zhang J, Malcolm D, Hislop-Jambrich J, Thomas CDL, Nielsen PMF. An anatomical region-based statistical shape model of the human femur. Comput Methods Biomech Biomed Eng Imaging Vis. 2014 Jul 3;2(3):176-85.

65. Bah MT, Shi J, Browne M, Suchier Y, Lefebvre F, Young P, King L, Dunlop DG, Heller MO. Exploring inter-subject anatomic variability using a population of patient-specific femurs and a statistical shape and intensity model. Med Eng Phys. 2015 Oct 1;37(10):9951007.

66. Väänänen SP, Isaksson H, Waarsing JH, Zadpoor AA, Jurvelin JS, Weinans H. Estimation of 3D rotation of femur in 2D hip radiographs. J Biomech. 2012 Aug 31;45(13):2279-83.

67. Ambellan F, Lamecker H, von Tycowicz C, Zachow S. Statistical shape models: understanding and mastering variation in anatomy. In: Rea PM, editor. Biomedical Visualisation: Volume 3. Cham: Springer International Publishing; 2019. p. 67-84. (Advances in Experimental Medicine and Biology).

Publisher's Note Springer Nature remains neutral with regard to jurisdictional claims in published maps and institutional affiliations. 